\title{
The fourth trimester: toward improved postpartum health and healthcare of mothers and their families in the United States
}

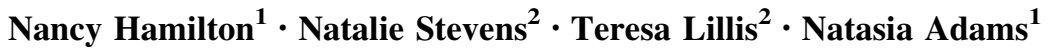

Published online: October 9, 2018

(C) Springer Science+Business Media, LLC, part of Springer Nature 2018

The United States (US) has the worst maternal mortality rate in the developed world. Moreover, it is one of the only countries in which the rates of life-threatening complications of childbirth have steadily increased during the past two decades. At an absolute level, maternal mortality is rare. Less than one-tenth of one percent $(17.3 \times 100,000)$ of women die from a pregnancy or childbirth-related problem. However, this statistic tells only part of the story. The maternal death rate of women of color is three to four times the death rate of White women. The number of women who attempt suicide within a year of giving birth is two to three times the number of maternal deaths. And, according to the Centers for Disease Control and Prevention (CDC, 2017), for every maternal death it is estimated that 50 or more women suffer serious medical complications. The breadth and impact of maternal morbidity and mortality is deeply disturbing for any society with access to the most advanced medicine in the world. Perhaps most disturbing is that the majority of these deaths and "nearmisses" are preventable. Thankfully maternal health experts are beginning to change their thinking about postpartum health. With this timely issue in mind, our aim in preparing this Special Issue was to present the latest research on health issues that affect women during the

A special thanks to Lauren Boddy, M.A., and Natasia Adams, M.A. for their help with this special issue.

Nancy Hamilton

nancyh@ku.edu

1 Department of Psychology, University of Kansas, 1416 Jayhawk Blvd, Lawrence, KS 66045, USA

2 Department of Behavioral Sciences, Rush Medical College, Chicago, IL, USA postpartum period and what we, as clinical scientists and behavioral medicine clinicians, might do fix the problem.

Before reading the articles in this Special Issue, we believe it would be helpful to have an epidemiological overview of birth in the United States, which sets the stage for examining maternal health during the postpartum period. For consistency we define the postpartum period as the time from birth to 6 months post-birth (Romano et al., 2010). The CDC recorded nearly four million $(3,978,497)$ births in 2015, the latest year for which there are complete epidemiological data (Martin et al., 2017). In 2015, the fertility rate reached a 10-year low (62.5 births per 1000 women), having steadily declined since 2005 . The average age of first-time mothers was 26.4, an all-time high. Within the broader statistic of fertility and birth rates, there are clear cohort specific trends. For teens, the birthrate has dropped dramatically over the past 25 years, having declined 46\% since 2007, and 64\% since 1991. Downward trends in birthrates were less dramatic for women in their 20 s, while birthrates for women in their 30s and 40s have been steadily increasing since the mid 1960s. Cesarean delivery rates have declined to $32 \%$ of births from their peak in 2009 but remain higher in the United States than in most other developed countries (Martin et al., 2017; OECD, 2017a). The rate of preterm births (defined as birth before 37 completed weeks of pregnancy) was nearly 1 in 10 of all births (9.63\%), a slight increase from 2014 and an interruption in a steady downward trend since 2005. To summarize, the population of mothers in the United States has been increasing in age for the past 30 years. More recent trends show that American mothers are increasingly likely to have a premature newborn and about one-third of American mothers are recovering from a cesarean delivery while caring for a newborn. The postpartum period is therefore bio-psychosocially complex and risky, consider- 
ing the epidemiological trends of increasing maternal age and high rates of prematurity and surgical delivery.

In contrast with the US, maternal mortality has declined in the last 25 years in all developed countries except the United States (Martin et al., 2017; Organisation for Economic Co-operation and Development, 2017b). The overall global decline in maternal mortality suggests that other developed countries may engage in practices that have effectively mitigated postpartum risk factors associated with maternal mortality and severe maternal morbidity. For example, there are obvious significant macro-level differences between the United States and other developed countries, such as the lack of universal access to prenatal health care in the US, which undoubtedly contributes to disparate trends in health outcomes. However, there are other specific obstetric practices that may reduce mortality and morbidity risk. For instance, a longer length of hospital stay (LOS) after delivery is associated with lower risk of maternal/infant death (Campbell et al., 2016; OECD, 2017c). In the United States, the average LOS after a singleton vaginal birth is only 1-2 days or $24-48 \mathrm{~h}$ after delivery. Although varied, European (e.g., Finland, United Kingdom), Middle Eastern (e.g., Israel, Turkey), and Asian (e.g., Japan) countries have longer LOS, ranging from 3.9 days (Turkey) to 16.5 days (Japan) with an average of 6.81 days for a singleton vaginal birth (Martin et al., 2017; OECD, 2017c). Additionally, cesarean-sections-another postpartum risk factor for maternal mortality and morbidity with population levels above $15 \%$ associated with worse outcomes (World Health Organization, 2015a, b) - has been reported to be lower in other developed countries, particularly in northern European countries (i.e., Finland, Norway, Iceland, and Sweden: around 15\%) and the United Kingdom (about 26\%), than the $31.9 \%$ reported in the US (Martin et al., 2017; OECD, 2017a). We recognize that post-birth hospital LOS and cesarean-sections represent only two risk factors associated with increased maternal mortality and morbidity and that a comprehensive analysis of risk factors is beyond the scope of this Special Issue. Nevertheless, the relatively high rates of surgical intervention alongside minimal structured medical support for newly-delivered mothers highlights some significant gaps in perinatal care that if addressed, may improve pregnancy survivorship, without radical changes to the US health care system.

Related to these broader health care trends, there is a growing public interest and concern regarding maternal morbidity and mortality in the United States. From 2015 to 2018, National Public Radio (NPR) and ProPublica published an award-winning series of articles in medical journalism. The series, "Lost Mothers", focused on maternal mortality and the failures of the US healthcare system to prevent these tragic losses. The release of this series has already spurred legislative action in multiple states (e.g., New Jersey, Texas) and at the federal level to shed light on and begin to fix this devastating public health problem. In June, the Senate Appropriations Committee voted to request $\$ 50$ million in new funding for a constellation of programs designed to reduce maternal mortality (Martin, 2018). Similarly, in July the Senate Committee on Health, Education, Labor, and Pensions passed the Maternal Health Accountability Act (S.112), a bill introduced by Senators Heidi Heitkamp (D., ND) and Shelley Moore Capito (R, WV) that would provide funding to establish state level maternal mortality review committees and establish mandatory reporting requirements for all pregnancy related deaths (2017). Although neither of these funding measures has yet to be passed, the media attention to the problem of maternal mortality has unquestionably had a legislative impact.

\section{From medical journalism to clinical science}

The stories of the "Lost Mothers" reveal the personified, emotional narrative that is left out of maternal mortality and morbidity statistics (ProPublica, 2018). These stories of loss are powerful and evoke images that are as difficult to forget as they are to imagine. For many families, these stories are marked by a tragic loss of life during a time when a new life was just beginning. It can be challenging to translate epidemiological data into human narrative and back again, but it is worth the exercise in an effort to grasp the scale and scope of new motherhood in the United States, where every year:

- Hundreds of women who give birth do not survive their baby's first 3 months of life.

- Hundreds of women who give birth attempt suicide in their baby's first year of life.

- Thousands of women experience major medical complications including organ failure, brain damage, excessive bleeding, loss of reproductive organs, and infertility.

- Hundreds of thousands of women suffer from debilitating depression and anxiety.

The "Lost Mothers" series illustrates, through compelling statistics and powerful narratives, how women who have just given birth are arguably the most underserved population in the US healthcare system. Indeed, maternal health experts now seem to recognize that preventive healthcare for women following childbirth is virtually nonexistent. Preventive healthcare in the postpartum period is almost exclusively focused on the newborn/infant. 
Although critically important, the American Academy of Pediatrics' (AAP) recommended schedule of eight wellbaby visits in the first year of an infant's life do not include formal evaluation of the person(s) considered most essential to the infant's welfare (i.e., the parent/s). By definition, pediatrics only peripherally considers the role of parents, who are themselves caregivers and not patients. Meanwhile, postpartum women's healthcare providers are tasked with the impossible challenge of addressing any number and degree of complexity of postpartum health concerns (e.g., abnormal bleeding, post-surgical recovery, pain, birth control, breastfeeding, sexual concerns, infection prevention, to name a few) in one 15-min postpartum visit 6 weeks after birth, of which only $40 \%$ of women actually attend, and for which providers are seldom reimbursed by insurance (American College of Obstetrics and Gynecology, 2018). Behavioral health is notably lacking in the vast majority of these routine visits. Except in cases of serious medical complications, for most women there is a vast lacuna in healthcare services between the end of one pregnancy and the beginning of the next (if there is a next pregnancy). This is an alarming healthcare gap occurring at a critical juncture in the course of human development. ACOG as recently as April 2018 updated their recommendations for postpartum healthcare, suggesting that providers see patients sooner after the birth of a baby and more frequently during the postpartum period if needed. The 2018 ACOG recommendation, released a few short months prior to this Special Issue, marks a crucial first step toward filling the harmful void in healthcare that leaves women largely unacknowledged.

\section{Next steps: toward comprehensive postpartum healthcare}

The ACOG (2018) committee recommendation to improve postpartum care merely scratches the surface in outlining the type of care and support that is needed to fill the healthcare void and quite literally save lives. Women (and their families) in the postpartum period need a strong web of support that combines medicine, social and family support, and mental health services. Once these factors are in place, tailored to and informed by the populations they serve, we will be better able to detect women who are most at risk of poor outcomes, optimize outcomes for women who are at lower risk, and in perhaps a small number of cases, intervene to prevent irreversible tragedy.

Before we set about to build this web of support, there are still many questions facing us as behavioral medicine specialists. What do we do next? What risk factors are necessary to address in order to prevent the worst outcomes? What risk factors might we be overlooking? Which interventions are most effective and for whom? What role do we play in preventive postpartum health care? How do we address issues of geographic, cultural, and economic barriers to provide services for those who need intervention? How can we also focus on promoting optimal wellbeing adjustment following childbirth, not just preventing fatalities? The following sections are intended to provide a framework for the articles contained in this Special Issue that address some of these important questions, while recognizing that there are important topics and populations we were unable to address.

Because this is the Journal of Behavioral Medicine and given that "the most common complication of childbirth" is postpartum depression (PPD; McCall-Hosenfeld et al., 2016; O'hara \& Swain, 1996; Wisner et al., 2013), studies related to PPD are strongly represented in this issue. However, our goal is for readers to come away from this compendium with an appreciation of a range of biomedical, psychological, and social processes underlying maternal health and complications of childbirth, as well as empirically-supported tools for their own clinical and public health initiatives.

\section{Special issue highlights}

The Special Issue opens with research focusing on strategies to shape routine health care and increase intervention access for women during the postpartum period. Despite the preponderance of data demonstrating efficacy and effectiveness of screening and treatment of postpartum depression and anxiety, there is a surprising lack of research examining how best to identify and meet women's needs for preventive and therapeutic support after childbirth. Verbiest and colleagues (2018) describe in their report how recent programs such as the Patient-Centered Outcomes Research Initiative (PCORI) have been successfully employed to fill this gap and to "elevate women's voices" in the shaping of postpartum health care. Walker and colleagues (2018) tackle the need to expand intervention dissemination with the creation of a "Mother-Centered Toolkit" while Geller and colleagues (2018) outline intensive day-programming for postpartum distress that addresses not just the mother's well-being but the oft-neglected mother-infant dyad as a system. Ayers and colleagues' paper (2018) addresses barriers to costeffectiveness in their randomized trial of an expressive writing intervention that may be more easily implemented in the context of Briton's National Health Care Service (NHS), a system that includes a postpartum home visit by a midwife as routine postpartum care. These studies are by no means a blueprint for an entire US postpartum healthcare overhaul, but they nevertheless offer researchers, 
clinicians, and women's health leaders with fruitful directions for feasible, cost-effective program ideas with the broadest applications for postpartum women.

Whereas the first set of papers in this Special Issue uses a broad lens to examine possible directions for postpartum healthcare, the next set of papers provides much-needed focus on mental health treatment of vulnerable populations. Indeed, the statistics cited at the beginning of this introduction shed light on one of the more distressing truths about postpartum health in the United States, namely, that women of color experience adverse outcomes on nearly every measure of postpartum health at shockingly disproportionate rates when compared to Whites. For example, in 2017 the maternal mortality rate for African American women was 43.5 per 100,000 (CDC, 2017), making it theoretically safer for an African American women to cross the border to give birth in Mexico (38 deaths per 100,000) or Canada (7 per 100,000) (World Health Organization, 2015a, b). Thus, any improvements, expansions, and changes to postpartum mental health treatment must be made with these health disparities in mind. This means more intervention research focused on effectiveness and generalizability of treatments for disadvantaged women, as described in Stevens and colleagues paper (2018), which uses an intersectionality-based intervention approach. Eliminating postpartum health disparities also means tailoring empirically-supported treatments to settings not routinely-used in provision of "traditional" mental health treatment, such as Tandon and colleagues' (2018) homevisiting model to deliver cognitive-behavioral therapy (CBT) in an urban-metro area. Finally, we recognize that rural women are also a dangerously underserved population due to the inadequate number of care providers who serve rural regions. Data extracted from CDC reports show that maternal morality is far higher in rural-America (29.4 per $100,000)$ than for the population of women as a whole (Maron, 2017) and that fewer than 50\% of rural women live within $30 \mathrm{~min}$ of a hospital with obstetric care (ACOG, 2018). Jacobson and colleagues address some of the care needs of both rural and Spanish-speaking women in "Pioneer Baby" (2018) while Guardino et al.'s (2018) findings highlight important considerations for evaluating levels of postpartum physical activity and exercise among low-income mothers in rural versus urban environments. These papers emphasize the importance of considering contextual and cultural differences when designing interventions.

As noted, this issue includes a number of papers that are focused on identifying risk and protective factors for PPD. Accortt and colleagues' (2018) highlight the roles of Vitamin D deficiency and antenatal depression symptoms in pregnancy as risk factors for adverse perinatal outcomes while Goyal and colleagues (2018) address the relationship between seasonal length of day during late gestation and delivery with postpartum depressive symptomology. Okun and colleagues (2018) demonstrate the role that poor sleep quality plays in predicting postpartum depression symptoms, even after controlling for antenatal mood symptoms. Finally, Cheadle et al. (2018) addressed the protective factors underlying the relationship between religiosity and spirituality with lower depressive symptoms in the postpartum. These methodologically strong papers offer a unique perspective on the longitudinal course of depressive symptoms across the perinatal period.

The final set of contributions to this Special Issue reflect the important role of interpersonal relationships in postpartum maternal health. Ross and colleagues paper (2018) investigate the interplay of partner satisfaction and conflict as a predictor of postpartum maternal cardiometabolic risk while Lillis et al. (2018) address the impact of daily positive and negative social interactions on maternal mood and sleep quality. A noteworthy finding regarding social relationships in the postpartum was the role of non-kin related relationships in Lillis and colleagues paper. Mothers in Lillis et al.'s paper reported at least as much contact with friends as they did with family members and rated those friend-related interactions as more enjoyable than those with their family. Although there is a pressing need to change OBGYN practices in order minimize acute risk of maternal morbidity and mortality, strengthening the web of support includes attending to social relationships and their influence on maternal health and well-being.

\section{Conclusion}

As clinical scientists we have a vital role in answering the public's "call to action" to reverse the upward trend of morbidity and mortality for this vulnerable and underserved population of women. In the context of this Special Issue, we have attempted to present a biopsychosocial approach to postpartum health. However, there are a number of notable limitations in our selection of papers. Although some of our papers focus on or at least include a race/ethnic/SES diverse sample, the preponderance of postpartum women represented in our data are White nonHispanic. Moreover, women in our study are predominantly heterosexual, or at least not identified as LBTQ, in part because there is still a paucity of data on lesbianidentified/same-sex families. Nevertheless, we hope that this selection of papers draws attention to the lives of new mothers and the health concerns that they may face during their first year of motherhood.

Despite these notable limitations, these papers suggest ways that postpartum care can better address the needs of mothers in the United States. As will be apparent from the 
papers in this Special Issue, postpartum care could be improved by integrating behavioral medicine into postpartum primary care. The 2018 recommendations by ACOG to increase postpartum medical care are a good start and are necessary to identify the emergent medical problems that have taken the lives of so many US mothers. However, the needs of postpartum mothers typically extend beyond medical care and are often more appropriately addressed by an interprofessional team of physicians, health psychologists, professional counselors, nurses, lactation consultants, social workers, physical therapists, and community health workers. In the context of an interprofessional team, postpartum care would ideally include screening for depression and other mental health concerns (e.g., anxiety, PTSD), onsite mental health services, counseling support for maternal-infant bonding, behavioral health consultation for weight management, a lactation consultant for nursing mothers, physical therapy for common physical problems such as incontinence and diastasis, and a sleep specialist who is attuned to sleep problems and solutions during the postpartum. This interprofessional model of care would represent a sea-change in standards of healthcare services for American mothers the postpartum period.

\section{Compliance with ethical standards}

Conflict of interest Nancy Hamilton, Natalie Stevens, Teresa Lillis, and Natasia Adams declare that they have no conflict of interest.

Human and animal rights This article does not contain any studies with human participants or animals performed by any of the authors.

\section{References}

American College of Obstetrics and Gynecology. (2018). Optimizing postpartum care. The American College of Obstetricians and Gynecologists, 131, e140-150.

Accortt, E. E., Lamb, A., Mirocha, J., \& Hobel, C. J. (2018). Vitamin $\mathrm{D}$ deficiency and depressive symptoms in pregnancy are associated with adverse perinatal outcomes. Journal of Behavioral Medicine. https://doi.org/10.1007/s10865-018-9924-9.

Ayers, S., Crawley, R., Button, S., Thornton, A., Field, A. P., Flood, C., et al. (2018). Evaluation of expressive writing for postpartum health: A randomised controlled trial. Journal of Behavioral Medicine. https://doi.org/10.1007/s10865-018-9970-3.

Campbell, O. M. R., Cegolon, L., Macleod, D., \& Benova, L. (2016). Length of stay after childbirth in 92 countries and associated factors in 30 low- and middle-income countries: Compilation of reported data and a cross-sectional analysis from nationally representative surveys. PLoS Medicine, 13, 1-24. https://doi.org/ 10.1371/journal.pmed.1001972

Centers for Disease Control and Prevention. (2017). Pregnancy mortality surveillance system. Retrieved January 15, 2018 from https://www.cdc.gov/reproductivehealth/maternalinfanthealth/ pmss.htm.

Cheadle, A. C. D., Dunkel Schetter, C., \& Community Child Health Network (CCHN). (2018). Mastery, self-esteem, and optimism mediate the link between religiousness and spirituality and postpartum depression. Journal of Behavioral Medicine. https:// doi.org/10.1007/s10865-018-9941-8.

Geller, P. A., Posmontier, B., Horowitz, J. A., Bonacquisti, A., \& Chiarello, L. A. (2018). Introducing mother baby connections: A model of intensive perinatal mental health outpatient programming. Journal of Behavioral Medicine. https://doi.org/10.1007/ s10865-018-9974-z.

Guardino, C. M., Hobel, C. J., Shalowitz, M. U., Ramey, S. L., Dunkel Schetter, C., \& Community Child Health Network (CCHN). (2018). Psychosocial and demographic predictors of postpartum physical activity. Journal of Behavioral Medicine. https://doi.org/10.1007/s10865-018-9931-x.

Goyal, D., Gay, C., Torres, R., \& Lee, K. (2018). Shortening day length: A potential risk factor for perinatal depression. Journal of Behavioral Medicine. https://doi.org/10.1007/s10865-018-99712.

Jacobson, L. T., Zackula, R., Redmond, M. L., Duong, J., \& Collins, T. C. (2018). Pioneer baby: Suggestions for pre- and postnatal health promotion programs from rural English and Spanishspeaking pregnant and postpartum women. Journal of Behavioral Medicine. https://doi.org/10.1007/s10865-018-9930-y.

Lillis, T. A., Hamilton, N. A., Pressman, S. D., Ziadni, M. S., Khou, C. S., Boddy, L. E., \& Wagner, L. M. (2018). Sleep quality buffers the effects of negative social interactions on maternal mood in the 3-6 month postpartum period: A daily diary study. Journal of Behavioral Medicine. https://doi.org/10.1007/s10865018-9967-y.

Maron, D. F. (2017). Maternal health care is disappearing in rural America. In Scientific American. Retrieved July 31, 2018 from https://www.scientificamerican.com/article/maternal-healthcare-is-disappearing-in-rural-america/.

Martin, J. A., Hamilton, B. E., Osterman, M. J. K., Driscoll, A. K., \& Mathews, T. J. (2017). Births: Final data for 2015. National Vital Statistics Reports, 66, 1-70.

Martin, N. (2018). U.S. Senate committee proposes $\$ 50$ million to prevent mothers dying in childbirth. In ProPublica. Retrieved August 10, 2018 from https://www.propublica.org/article/ussenate-committee-maternal-mortality-prevention-proposal.

Maternal Health Accountability Act. (2017). S. B. 1112, Senate (2017). Retrieved August 10, 2018 from https://www.congress. gov/115/bills/s1112/BILLS-115s1112is.pdf.

McCall-Hosenfeld, J. S., Phiri, K., Schaefer, E., Zhu, J., \& Kjerulff, K. (2016). Trajectories of depressive symptoms throughout the peri- and postpartum period: Results from the first baby study. Journal of Women's Health, 25, 1112-1121.

O'hara, M. W., \& Swain, A. M. (1996). Rates and risk of postpartum depression-a meta-analysis. International Review of Psychiatry, 8, 37-54. https://doi.org/10.3109/09540269609037816

Okun, M. L., Mancuso, R. A., Hobel, C. J., Schetter, C. D., \& Coussons-Read, M. (2018). Poor sleep quality increases symptoms of depression and anxiety in postpartum women. Journal of Behavioral Medicine. https://doi.org/10.1007/s10865-018-9950-7.

Organisation for Economic Co-operation and Development. (2017a). Caesarean sections [Data file]. http://doi.org/10.1787/adc3c39fen

Organisation for Economic Co-operation and Development. (2017b). Health status: Maternal and infant mortality [Data file]. Retrieved July 16, 2018 from https://stats.oecd.org/index. aspx?queryid=30116.

Organisation for Economic Co-operation and Development. (2017c). Length of hospital stay [Data file]. http://doi.org/10.1787/ 8dda6b7a-en

Pro Publica (2018). Lost mothers: Maternal care and preventable deaths. Retrieved January 10, 2018 from https://www. propublica.org/series/lost-mothers. 
Romano, M., Cacciatore, A., Giordano, R., \& La Rosa, B. (2010). Postpartum period: Three distinct but continuous phases. Journal of Prenatal Medicine, 4, 22-25.

Ross, K. M., Guardino, C., Hobel, C. J., Dunkel Schetter, C. (2018). Partner relationship satisfaction, partner conflict, and maternal cardio-metabolic health in the year following the birth of a child. Journal of Behavioral Medicine. https://doi.org/10.1007/s10865018-9947-2.

Stevens, N. R., Heath, N. M., Lillis, T. A., McMinn, K., Tirone, V., \& Sha'ini, M. (2018). Examining the effectiveness of a coordinated perinatal mental health care model using an intersectionalfeminist perspective. Journal of Behavioral Medicine. https:// doi.org/10.1007/s10865-018-9973-0.

Tandon, S. D., Ward, E. A., Hamil, J. L., Jimenez, C., \& Carter, M. (2018). Perinatal depression prevention through home visitation: A cluster randomized trial of mothers and babies 1-on-1. Journal of Behavioral Medicine. https://doi.org/10.1007/s10865-0189934-7.

Verbiest, S., Tully, K., Simpson, M., \& Stuebe, A. (2018). Elevating mothers' voices: Recommendations for improved patient-centered postpartum. Journal of Behavioral Medicine. https://doi. org/10.1007/s10865-018-9961-4.
Walker, L. O., Sterling, B. S., Becker, H., Hendrickson, S., \& Xie, B. (2018). Development and evaluation of a mother-centered toolkit for postpartum behavioral and psychosocial health. Journal of Behavioral Medicine. https://doi.org/10.1007/s10865-018-99285.

Wisner, K. L., Sit, D. K. Y., McShea, M. C., Rizzo, D. M., Zoretich, R. A., Hughes, C. L., et al. (2013). Onset timing, thoughts of self-harm, and diagnoses in postpartum women with screenpositive depression findings. JAMA Psychiatry, 70, 490-498. https://doi.org/10.1001/jamapsychiatry.2013.87

World Health Organization. (2015a). Maternal mortality ratio (per 100,000 live births): 2015. Retrieved July 31, 2018 from http:// gamapserver.who.int/gho/interactive_charts/mdg5_mm/atlas. html.

World Health Orgranization. (2015b). WHO statement on caesarean section rates. World Health Organization. Retrieved July 31, 2018 from http://apps.who.int/iris/bitstream/handle/10665/ 161442/WHO_RHR_15.02_eng.pdf;jsessionid=C2F1DA7 A003528DD740C46FE44FAA40F? sequence $=1$. 